ARTICLE

\title{
Novel factors to predict respiratory viral disease progression in allogeneic hematopoietic cell transplant recipients
}

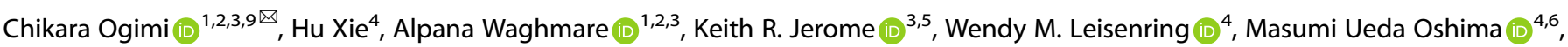
Paul A. Carpenter ${ }^{2,4,7}$, Janet A. Englund ${ }^{1,2}$ and Michael Boeckh ${ }^{3,4,8}$

(c) The Author(s), under exclusive licence to Springer Nature Limited 2022

We assessed novel factors and the immunodeficiency scoring index (ISI) to predict progression to lower respiratory tract infection (LRTI) among hematopoietic cell transplant (HCT) recipients presenting with upper respiratory tract infection (URTI) with 12 viruses in the PCR era. We retrospectively analyzed the first respiratory virus detected by multiplex PCR in allogeneic HCT recipients (4/ 2008-9/2018). We used Cox proportional hazards models to examine factors for progression to LRTI within 90 days among patients presenting with URTI. A total of 1027 patients ( 216 children and 811 adults) presented with URTI only. Among these, 189 (18\%) progressed to LRTI (median: 12 days). Multivariable models demonstrated a history of $>1$ transplant, age $\geq 40$ years, time post-HCT ( $\leq 30$ days), systemic steroids, hypoalbuminemia, hyperglycemia, cytopenia, and high ISI (scores 7-12) were associated with an increased risk of progression to LRTI. Respiratory syncytial virus and human metapneumovirus showed the highest progression risk. Patients with $\geq 3$ independent risk factors or high ISI scores were highly likely to progress to LRTI. We identified novel risk factors for progression to LRTI, including history of multiple transplants and hyperglycemia, suggesting an intervention opportunity with glycemic control. ISI and number of risk factors appear to predict disease progression across several viruses.

Bone Marrow Transplantation (2022) 57:649-657; https://doi.org/10.1038/s41409-022-01575-z

\section{INTRODUCTION}

The significant impact of viral lower respiratory tract infections (LRTI) on morbidity and mortality in hematopoietic cell transplant (HCT) recipients is now widely appreciated [1]. Risk factors for the development of LRTI for various viruses have been identified [1-3]. A scoring system, the immunodeficiency scoring index (ISI), was originally developed to predict risk of progression to LRTI in HCT recipients who present with respiratory syncytial virus (RSV) upper respiratory tract infection (URTI) without concomitant LRTI [4]. The score has been subsequently evaluated for different viruses in different cohorts [5-10]. However, most studies were relatively small, and importantly, included patients with LRTI present at initial evaluation, which differed from the original method. For clinical practice and design of early intervention strategies, assessments of risk factors for progression to LRTI at URTI stage are particularly relevant.

Hyperglycemia has been recognized as a prognostic factor for poor outcomes in patients with SARS-CoV-2 [11-13]; however, it has not been well studied as a risk factor for progression to LRTI in HCT recipients except recently for seasonal coronaviruses [14]. Simultaneously, repeated transplants have become more common but the impact of this on progression is unknown. Limited data exist regarding the relative risk of progression to LRTI by type of virus among patients presenting with URTI, especially in the molecular diagnostic era.
The objective of this study was to identify novel risk factors for progression to LRTI and examine the applicability of the ISI to other viruses in allogeneic HCT recipients presenting with viral URTI. We aimed to assess the probability of LRTI development by virus type and compare the performance of a simplified scoring system based on easily accessible criteria.

\section{METHODS \\ Study design}

We reviewed medical records from pediatric and adult allogeneic HCT recipients whose first respiratory viral infection was identified by molecular testing from upper respiratory tract samples post-HCT. Patients presenting with LRTI were excluded. Transplant recipients were identified from 2 separate cohorts at Fred Hutchinson Cancer Research Center (Fred Hutch). The first cohort included patients undergoing transplant between July 2009-September 2018 who had respiratory tract samples collected and tested for clinical purposes. Our standard of care is to perform multiplex PCR using upper respiratory tract samples in patients with respiratory symptoms. The second cohort was a subset of patients from a prospective surveillance study of allogeneic HCT recipients undergoing transplant from April 2008-February 2010 in which standardized respiratory symptom surveys and multiplex respiratory PCR tests were performed pre-HCT, weekly during the first 100 days post-HCT, and at least every 3 months through 1 year post-HCT [15]. Clinical samples were also collected in this group at clinicians' discretion if respiratory symptoms

\footnotetext{
${ }^{1}$ Pediatric Infectious Diseases Division, Seattle Children's Hospital, Seattle, WA, USA. ${ }^{2}$ Department of Pediatrics, University of Washington, Seattle, WA, USA. ${ }^{3}$ Vaccine and Infectious Disease Division, Fred Hutchinson Cancer Research Center, Seattle, WA, USA. ${ }^{4}$ Clinical Research Division, Fred Hutchinson Cancer Research Center, Seattle, WA, USA. ${ }^{5}$ Department of Laboratory Medicine and Pathology, University of Washington, Seattle, WA, USA. ${ }^{6}$ Division of Medical Oncology, University of Washington, Seattle, WA, USA. ${ }^{7}$ Pediatric Hematology Oncology, Seattle Children's Hospital, Seattle, WA, USA. ${ }^{8}$ Department of Medicine, University of Washington, Seattle, WA, USA. ${ }^{9}$ Present address: Pediatric Infectious Diseases, National Center for Child Health and Development, Tokyo, Japan. ${ }^{\aleph_{\text {email: }}}$ cogimi@fredhutch.org
}

Received: 27 September 2021 Revised: 18 December 2021 Accepted: 10 January 2022

Published online: 16 February 2022 
were noted. For the current study, we included only subjects with respiratory symptoms at the time of first respiratory virus detection from these cohorts. Demographic and clinical data were extracted from Fred Hutch's database and medical chart review. The study was approved by the Institutional Review Board at Fred Hutch.

\section{Laboratory testing}

Upper (nasal washes combined with throat swab or swab from nasal mid turbinate) and lower (bronchoalveolar lavage or lung biopsy) respiratory tract samples were tested by multiplex semi-quantitative, reverse transcription-PCR for 12 respiratory viruses [15-17]. All PCR reactions were performed according to College of American Pathologists standards.

\section{Definitions}

URTI was defined as virus detection in an upper respiratory tract sample with respiratory symptoms. Proven or probable LRTI was defined as having virus detected from a lower respiratory tract sample with or without new pulmonary infiltrates by chest radiography, respectively [18]. Possible LRTI was defined as having virus detected from an upper respiratory tract sample with new pulmonary infiltrates but without confirmation of virus in a lower respiratory tract sample. Patients who met criteria for LRTI within 1 day of URTI were considered to have LRTI at presentation and were excluded [19]. The index transplant was the first occurring during the study period. The time interval between transplants in patients was not restricted. A copathogen in blood was defined as a pathogen or antigen (bacteria, fungi, virus, galactomannan enzyme-linked immunosorbent assay) detected in blood samples obtained within 2 days of URTI diagnosis [18]. The most recent values of blood cell counts, highest serum glucose ( $>200 \mathrm{mg} / \mathrm{dL})$ and lowest serum albumin $(<=3 \mathrm{~g} / \mathrm{dL}$ ) values within 2 weeks before URTI were recorded [20]. Similarly, highest daily systemic steroid dose and use of oral budesonide and/or beclomethasone within 2 weeks prior to URTI were collected.

\section{Statistical analysis}

The probability of progression to LRTI among patients who presented with URTI was estimated by cumulative incidence curves, treating death as a competing risk. Gray's test compared cumulative incidence probabilities between categories. Fine and Gray versions of Cox proportional hazards models were used to estimate unadjusted and adjusted hazard ratios (HRs) for progression to LRTI within 90 days of URTI. Covariates with P values < 0.05 in univariable analyses were candidates for inclusion in the multivariable models unless variables deemed in similar causal pathways. ISI and components of ISI (e.g., steroid use, blood cell counts) were not included in the same models [4]. Separately, multivariable Cox regression models without ISI evaluated each risk factor candidate. Since serum glucose level and steroid dose are likely correlated, we also performed a sensitivity analysis using a composite variable for glucose value and steroid dose to evaluate joint effects of each level. Two-sided $P$ values $<0.05$ were considered statistically significant. All statistical analyses were performed using SAS 9.4 for Windows (SAS Institute, Cary, NC). Original data are available by request to the corresponding author.

\section{RESULTS}

\section{Patient characteristics and LRTI}

Among 2552 patients undergoing allogeneic HCT during the study period, 1027 patients ( 216 children and 811 adults) met the study inclusion criteria. Characteristics of each infection group by virus type are shown in Table 1 and Supplementary Table 1. Among 1027 patients who presented with URTI, human rhinovirus (HRV) was the most common virus detected, followed by parainfluenza viruses (PIV) and seasonal coronaviruses; 189 (18\%) progressed to LRTI within 90 days (median: 12 days, interquartile range: 6-24 days)(Fig. 1a). Figure $1 \mathrm{~b}$ shows the incidence rate of LRTI after first infection by each virus among 2552 allogeneic HCT recipients. These data also included patients who presented with LRTI. The highest incidence of LRTI was seen with HRV (6.2\%), followed by PIV (4.7\%) and RSV (3.6\%).

\section{History of multiple HCT}

Among 1,027 patients who presented with URTI, 157 (15\%) patients had a history of multiple HCTs, and 15 patients (1.5\%) had undergone $>=3$ transplants (Supplementary Table 2). NonHodgkin lymphoma (NHL) $(45 / 157,29 \%)$ and multiple myeloma $(52 / 157,33 \%)$ were the most common indications for second transplant. This reflects institutional practice and clinical trials open during the study period using tandem autologous transplant followed by non-myeloablative allogeneic transplant for high-risk multiple myeloma and NHL patients. The median interval between prior and recent HCT was 176 days with a range of 41-8001 days. Seventy-nine percent of patients with $>1$ HCT (124) received nonmyeloablative conditioning, and the majority (121) had myeloablative conditioning during prior HCTs. Among patients with multiple HCTs, the rate of progression to LRTI was $24 \%(23 / 97)$ in those with multiple myeloma or $\mathrm{NHL}$ and $28 \%(17 / 60)$ in those with other underlying diseases (Supplementary Table 3).

\section{ISI to predict progression to LRTI}

Variables evaluated in univariable Cox models to identify factors associated with progression to LRTI are shown in Supplementary Table 4. In multivariable models, history of $>1 \mathrm{HCT}$, hypoalbuminemia $(<=3 \mathrm{~g} / \mathrm{dL}$ ), hyperglycemia (highest glucose $>200 \mathrm{mg} / \mathrm{dL}$ ), high ISI (scores 7-12), RSV, and human metapneumovirus infections (compared to HRV) were associated with an increased risk of progression to LRTI (Fig. 2). COV was associated with a lower risk of progression. The risk of the outcome did not significantly differ between the moderate ISI group (scores 3-6) and the low ISI group (scores $0-2$ ). The cumulative incidence plots of progression to LRTI stratified by ISI overall and for each virus are shown in Fig. 3 and Supplementary Fig. 1. Overall, patients in the high ISI group appeared more likely to progress to LRTI across several viruses; trends for COV, human metapneumovirus, and multiple viruses were less clear.

\section{Risk factors and risk stratification for progression to LRTI}

We performed multivariable models without ISI by including candidate risk factors of ISI. Among variables significant in univariable models, we excluded variables potentially in similar causal pathways. Further description of the risk factors evaluated are included in Supplementary materials. Figure 4 shows the results of both multivariable models 1 and 2 for each cytopenia variable. Overall, results were similar in both models. Independent risk factors identified were age $>=40$ years, albumin $<=3 \mathrm{~g} / \mathrm{dL}$, a history of multiple HCT, systemic steroid use, early timing postHCT $\left(<=30\right.$ days), lymphopenia $\left(<=100 \times 10^{6}\right.$ cells/L) and/or monocytopenia $\left(<=100 \times 10^{6}\right.$ cells/L), and respiratory viruses other than COV. Highest glucose $>200 \mathrm{mg} / \mathrm{dL}$ was significant in model 2 with monocytopenia variable; the association was borderline in model 1 with lymphopenia variable $(p=0.051)$.

We then analyzed progression rates using monocytopenia (model 2), according to number of risk factors present at the URTI diagnosis for overall and each virus type (Fig. 3, Supplementary Fig. 1). Few patients progressed to LRTI in groups with zero or one risk factor, whereas higher progression rates were observed with $>=3$ risk factors, except for patients with multiple viruses where trends were unclear.

\section{Joint effects of glucose level and steroid dose on progression to LRTI}

Given the collinearity between glucose level and steroid dose, we performed a sensitivity multivariable analysis using a composite variable for glucose levels and steroid doses to evaluate both factors. As an adjustment factor, we chose lymphopenia $(<=$ $200 \times 10^{6}$ cells/L), a commonly used cutoff $[4,9,21]$. All combinations of glucose levels and steroid dose groups were associated with significantly higher hazard ratios compared to the group with no steroid and glucose $<=200 \mathrm{mg} / \mathrm{dL}$ except the group with steroid $>=1 \mathrm{mg} / \mathrm{kg}$ and glucose $>200 \mathrm{mg} / \mathrm{dL}$ (not significantly higher, $p=0.058$; Supplementary Fig. 2). We created cumulative incidence plots of progression to LRTI according to the highest 


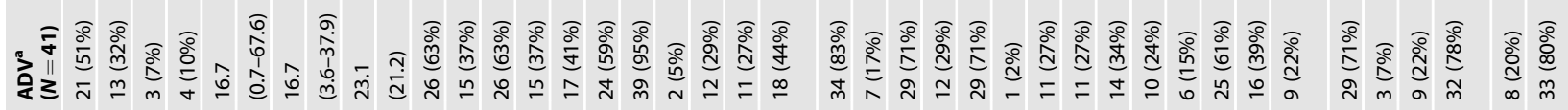

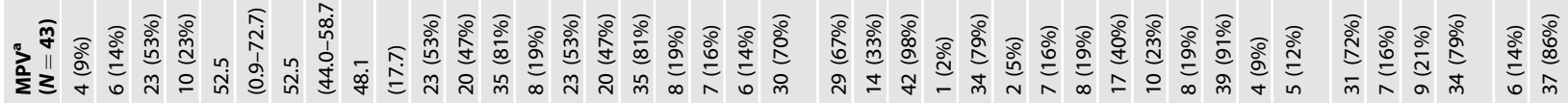

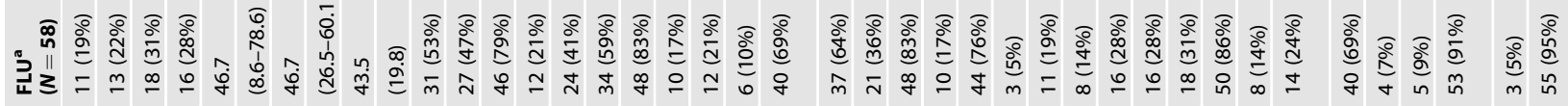

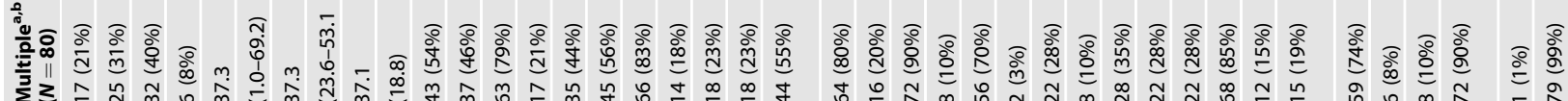

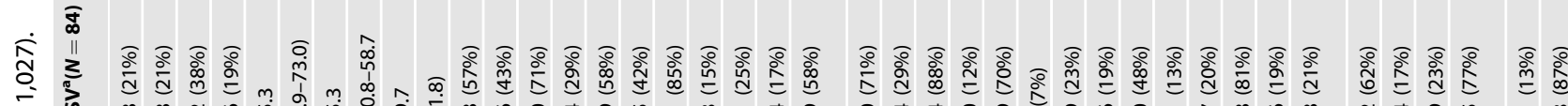
II z

ऽ

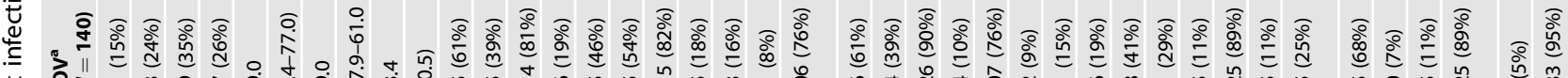

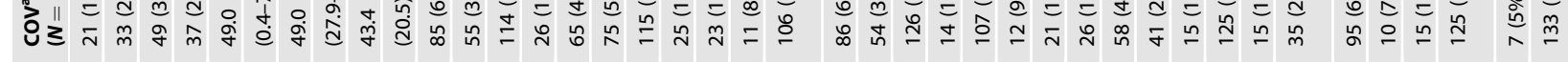

항

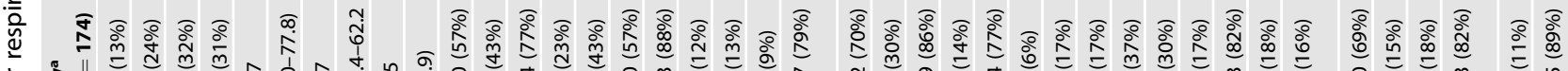

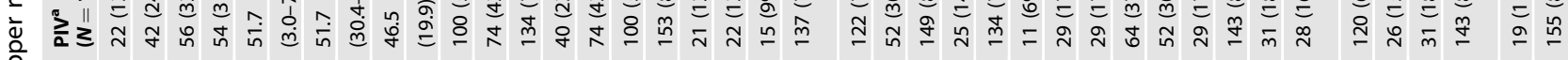
을

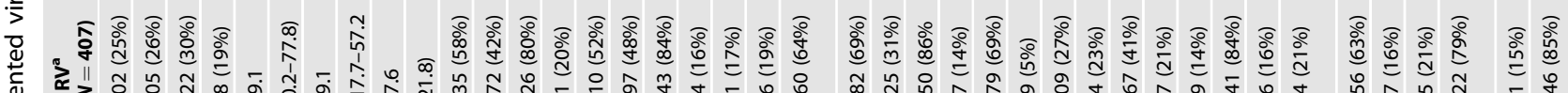

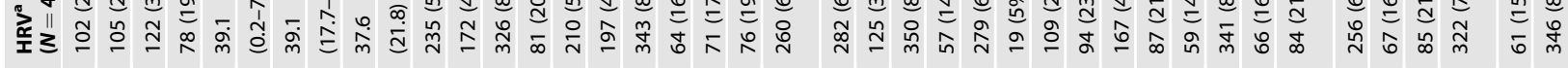

응

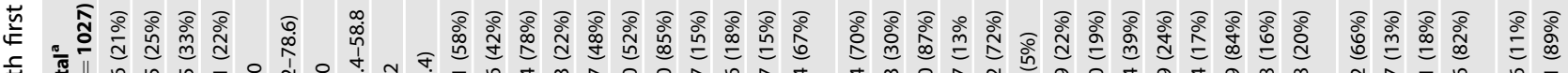

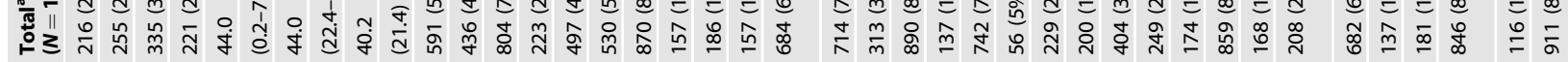

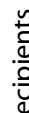

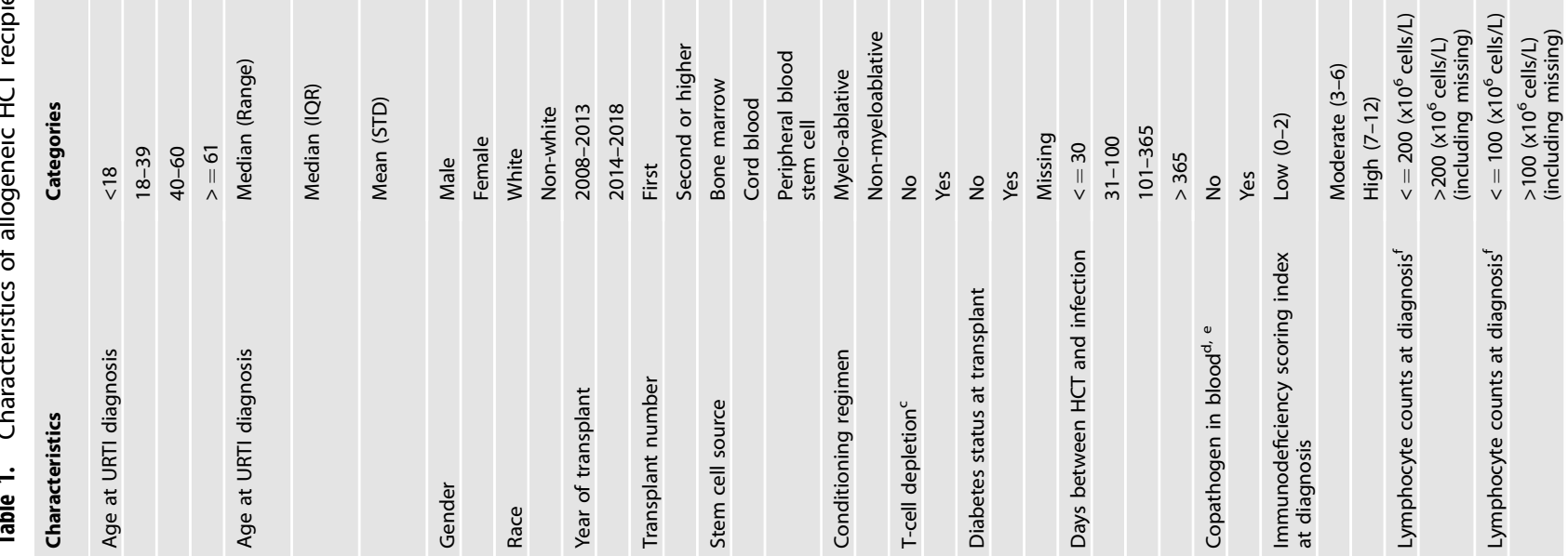




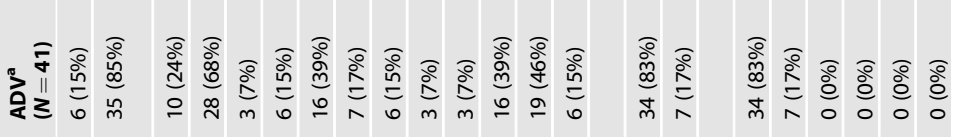

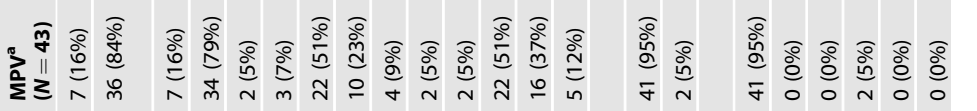

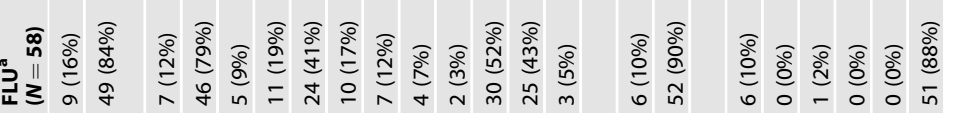

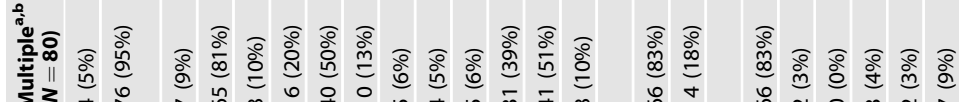

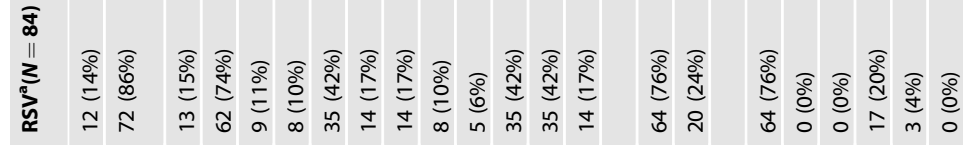

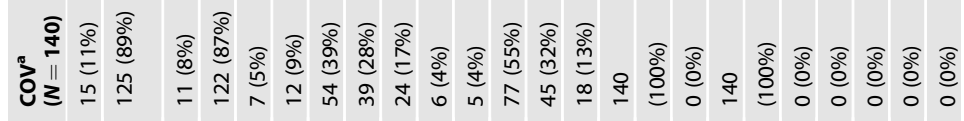

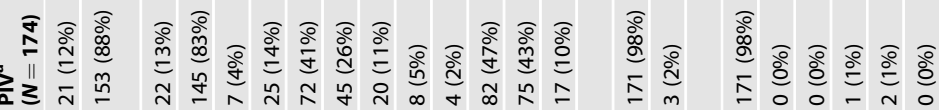

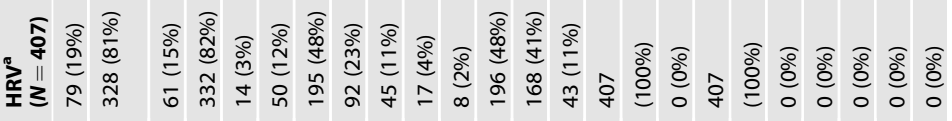

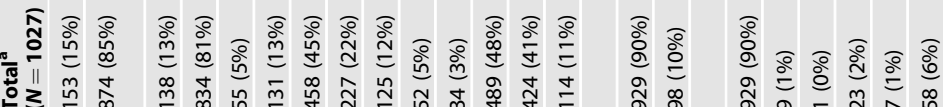

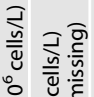

흫 ㅎํำ

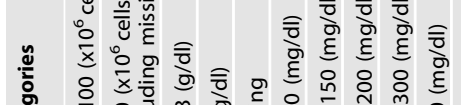

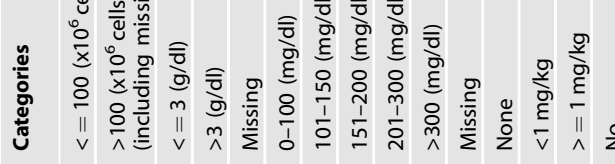

|
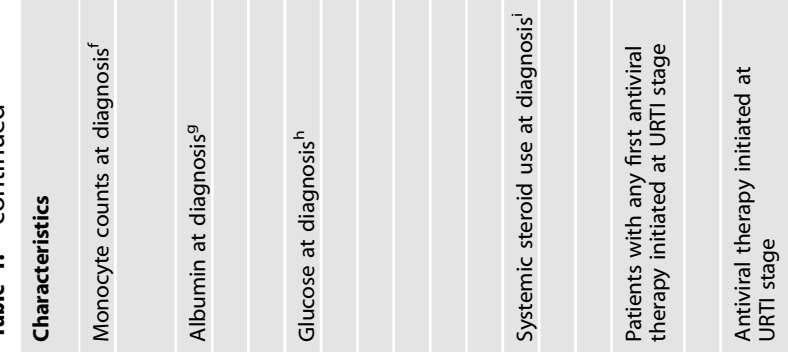

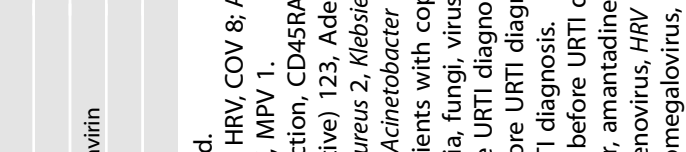

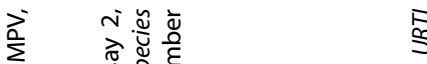

$\because \quad$ बै

䒕 艺

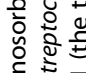

है

iे

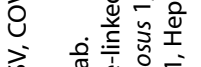

है ڤิ हิ

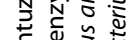

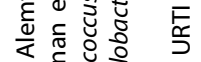

व

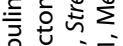

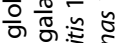

รั้ $气$ है के

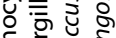

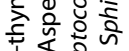

的焉=

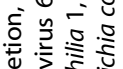

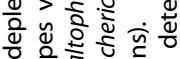

†े है

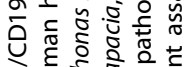

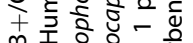

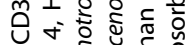

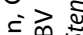

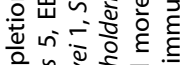

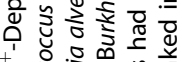

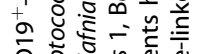

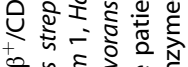

in कै है

वे

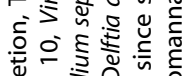

$\frac{0}{0}, \frac{n}{2}$

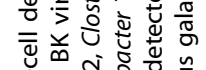

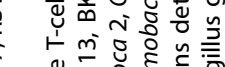

之.

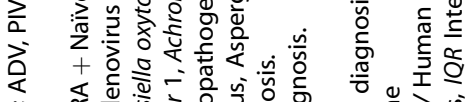

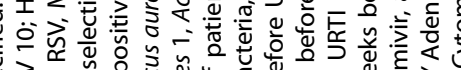


a

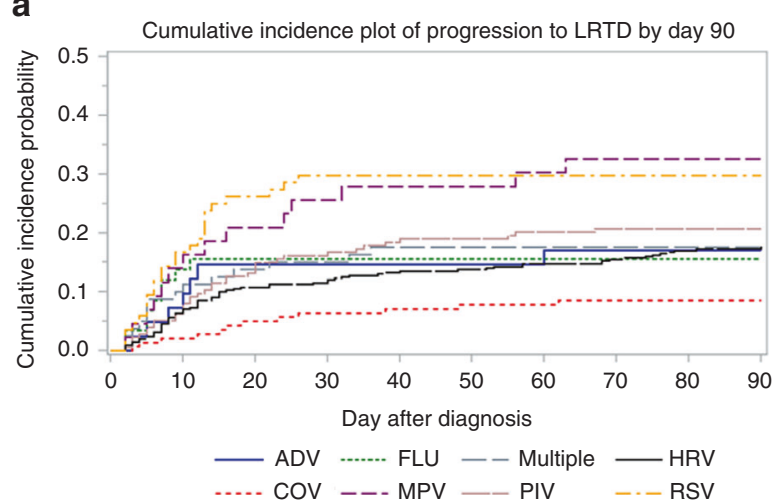

b

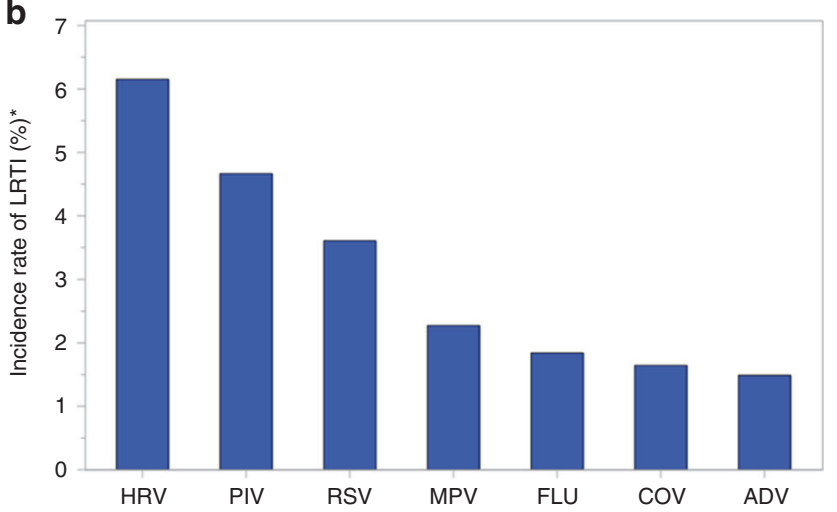

Fig. 1 Incidence of progression to LRTI and overall incidence of LRTI. a Cumulative incidence of progression to LRTI within 90 days among patients presenting with first viral URTI. b Incidence rate of LRTI after first infection by each virus among 2552 allogeneic HCT recipients. X axis indicates type of virus. *Only the LRTI developed from the first infection following allogeneic HCT was counted as an LRTI incident (Y axis). All cases of LRTI (presented with LRTI as well as presented with URTI and then progressed to LRTI) were counted as long as they were first infection by each virus type after transplant. LRTI Lower respiratory tract infection, URTI Upper respiratory tract infection, HCT Hematopoietic cell transplant, ADV Adenovirus, FLU Influenza, HRV Human rhinovirus, COV Seasonal coronavirus, MPV Human metapneumovirus, PIV Parainfluenza virus, RSV Respiratory syncytial virus.

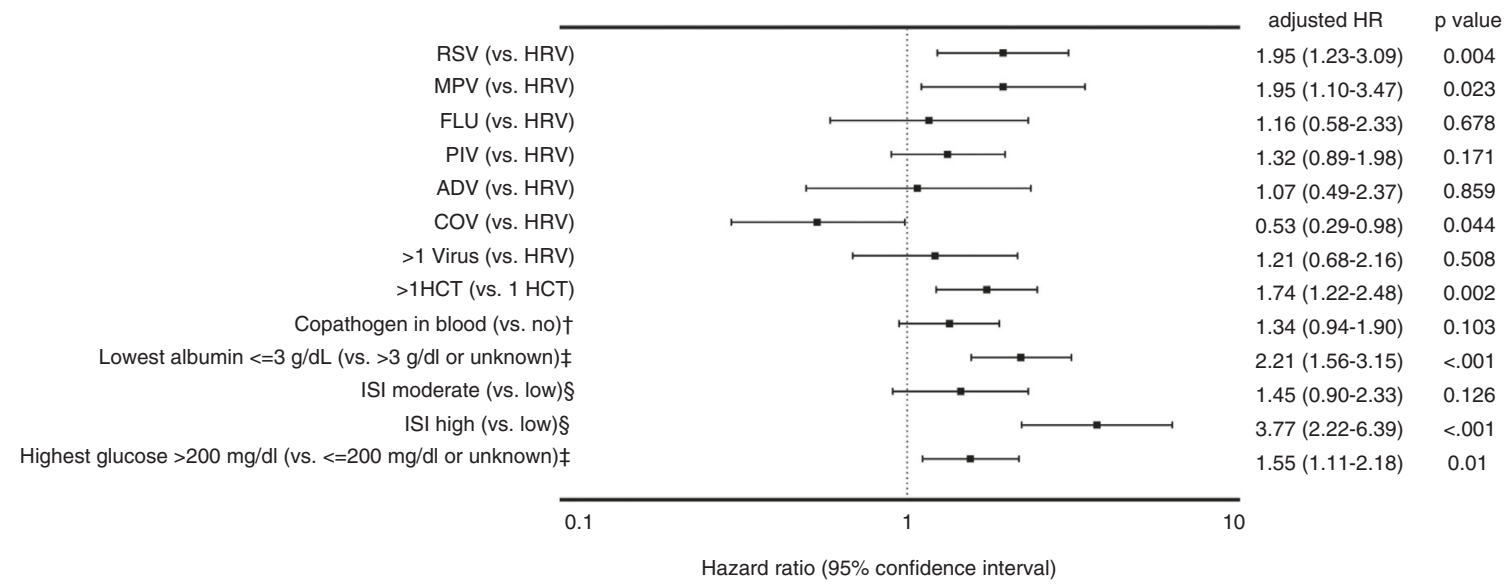

Fig. 2 Multivariable Cox proportional hazards models for progression to viral LRTI including immunodeficiency scoring index. ${ }^{\dagger}$ Defined as a pathogen or antigen (bacteria, fungi, virus, Aspergillus galactomannan enzyme-linked immunosorbent assay) detected in a blood within 2 days of upper respiratory tract infection diagnosis. ${ }^{*}$ Using values within 2 weeks before upper respiratory tract infection diagnosis. $\S$ at upper respiratory tract infection diagnosis. Components of immunodeficiency scoring index with assigned scores are as follows: absolute neutrophil count of less than $500 \times 10^{6}$ cells/L (3), absolute lymphocyte count of less than $200 \times 10^{6}$ cells/L (3), age of 40 years or greater (2), the use of a myeloablative conditioning regimen (1), any graft-versus-host disease (1), corticosteroids used within 30 days of respiratory viral infection (1), recent (within 30 days of infection) or pre engraftment allogeneic hematopoietic cell transplant (1). The patients are stratified into low (0-2), moderate (3-6), and high (7-12) risk groups. LRTI Lower respiratory tract infection, RSV Respiratory syncytial virus, HRV Human rhinoviruses, MPV Human metapneumovirus, FLU Influenza, PIV Parainfluenza viruses 1-4, ADV Adenovirus, COV Seasonal coronavirus, HCT Hematopoietic cell transplant, ISI Immunodeficiency scoring index.

glucose value with $200 \mathrm{mg} / \mathrm{dL}$ as a cut-off for each virus type (Supplementary Fig. 3). Groups with higher glucose progressed to LRTI at higher rates for HRV and PIV; cumulative incidence estimates reached higher values for the higher glucose group with other viruses but differences were not significant.

\section{DISCUSSION}

This study identified novel risk factors for viral disease progression to LRTI in allogeneic HCT recipients, including a history of multiple transplants and hyperglycemia, based on multivariable models. ISI appears to predict patients at risk for progression to LRTI for several viruses, including RSV, HRV, PIV and adenovirus. We also documented that patients with $>=3$ independent risk factors are highly likely to progress to LRTI.

Surprisingly, a history of multiple transplants has not been recognized as a risk factor for viral progression to LRTI or other serious infections in HCT recipients [14], although multiple transplants are becoming more common with newer conditioning regimens. Our finding intuitively makes sense, since those undergoing repeated transplants are subject to additional cumulative impacts of chemotherapy, radiation and conditioning. In our cohort, the majority of patients were adults who received a myeloablative conditioning regimen prior to non-myeloablative conditioning regimen. Patients with multiple myeloma or $\mathrm{NHL}$ often undergo tandem autologous HCT followed by nonmyeloablative allogeneic HCT. Since those patients tend to have intrinsic $B$ cell dysfunction or receive $B$ cell-targeted chemotherapy, we hypothesized those patients might have higher rates of LRTI progression given prolonged B-cell dysfunction. In fact, there was a higher proportion of multiple myeloma or $\mathrm{NHL}(97 / 157,62 \%)$ in patients with multiple HCTs than those with single HCT $(28 / 870$, $3 \%)$. However, among those with multiple HCTs, the rate of progression to LRTI appeared lower in patients with multiple 

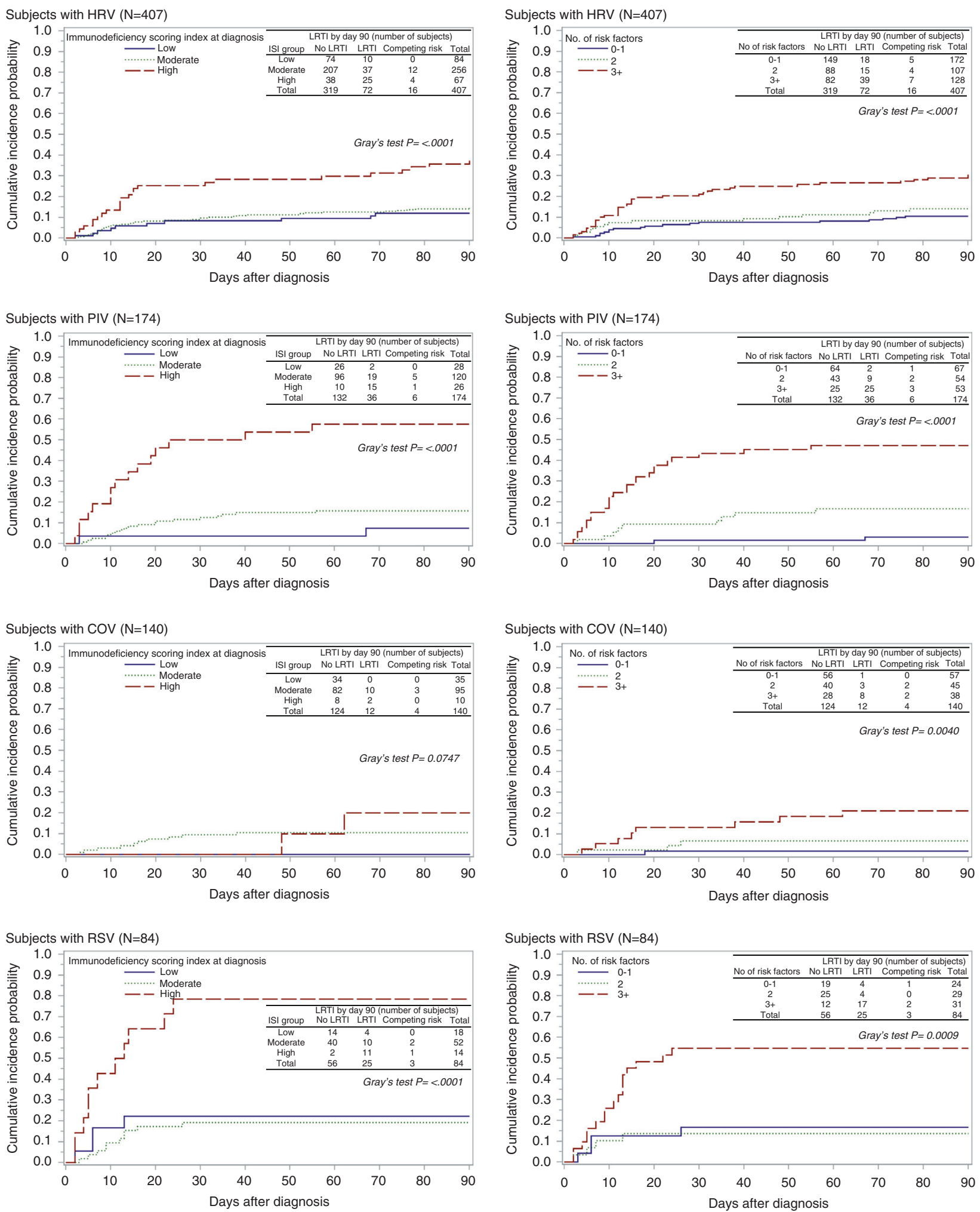

Fig. 3 Cumulative incidence of progression to LRTI within 90 days among patients presenting with URTI by virus type, stratified by immunodeficiency scoring index as well as number of risk factors. Gray's test was used to compare cumulative incidence probabilities between categories. Risk factors were age $>=40$ years, albumin $<=3 \mathrm{~g} / \mathrm{dL}$, a history of multiple HCT, systemic steroid use, early timing post$\mathrm{HCT}\left(<=30\right.$ days), monocytopenia $\left(<=100 \times 10^{6}\right.$ cells/L) and respiratory viruses other than COV. LRTI Lower respiratory tract infection, HRV Human rhinoviruses, PIV Parainfluenza viruses 1-4, COV Seasonal coronavirus, RSV Respiratory syncytial virus, ISI Immunodeficiency scoring index. 


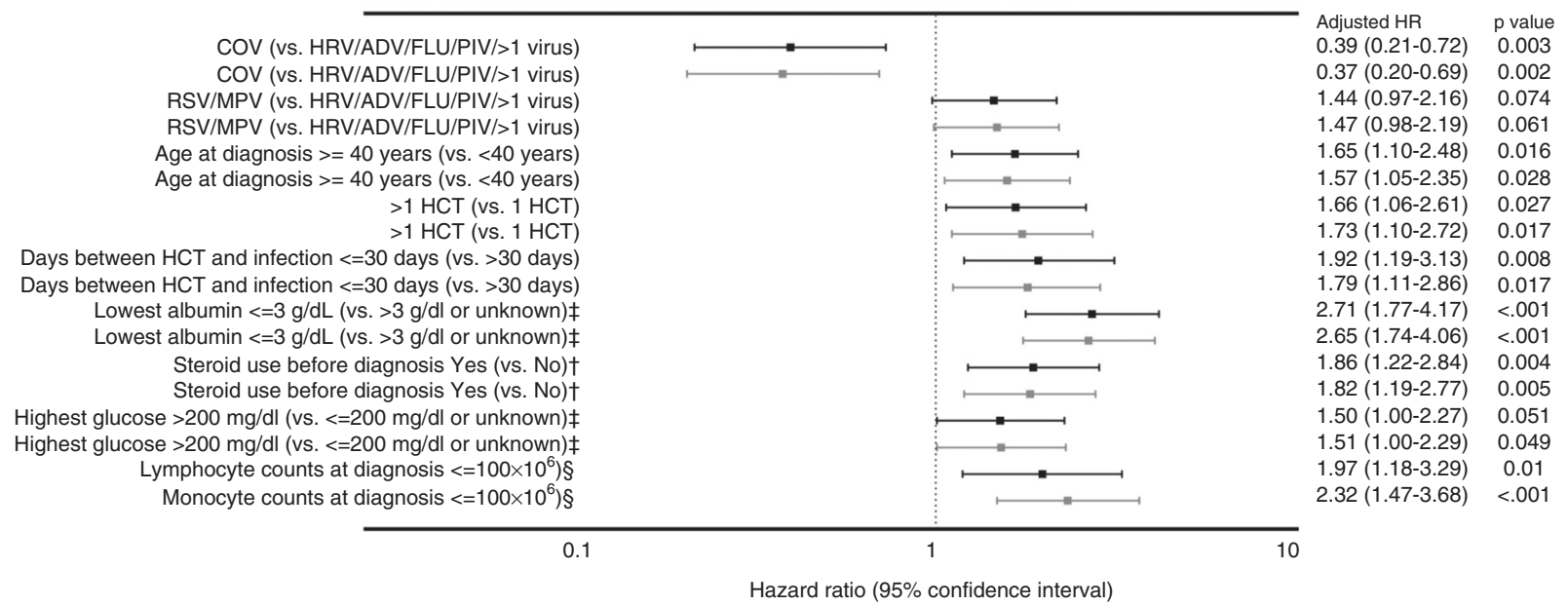

Adjusted, model 1 (lymphopenia)

Adjusted, model 2 (monocytopenia)

Fig. 4 Multivariable Cox proportional hazards models for progression to viral LRTI without immunodeficiency scoring index. Both adjusted models included same variables except for cytopenia variables (model 1 included lymphocyte counts and model 2 included monocyte counts). ${ }^{\ddagger}$ using values within 2 weeks before upper respiratory tract infection diagnosis. ${ }^{\dagger}$ Use of systemic steroids within 2 weeks before upper respiratory tract infection diagnosis. $\S$ using nearest value within 2 weeks before URTI diagnosis. LRTI Lower respiratory tract infection, COV Seasonal coronavirus, HRV Human rhinoviruses, ADV Adenovirus, FLU Influenza, PIV Parainfluenza viruses 1-4, RSV Respiratory syncytial virus, MPV Human metapneumovirus, HCT Hematopoietic cell transplant.

myeloma or $\mathrm{NHL}(23 / 97,24 \%)$ compared to those with other underlying diseases $(17 / 60,28 \%)$. Notably, the significance of the association remained after adjustments (age, cytopenia, and timing of infection), suggesting that some unrecognized elements may play a role. Further studies are warranted to explore this intriguing finding.

Less data exist regarding hyperglycemia for respiratory viral disease progression in immunocompromised hosts. Human and animal studies suggest that diabetes is a risk factor for severe influenza infections $[22,23]$. Hyperglycemia is a prognostic factor for poor outcomes in patients with SARS-CoV-2, regardless of history of diabetes [11-13], and recent studies indicated hyperglycemia with steroid use is a strong predictor of seasonal coronavirus disease progression in allogeneic HCT recipients [14]. The mechanism of hyperglycemia affecting innate immunity is not known [24-26]. Our multivariable models demonstrated a significant association between hyperglycemia and progression to LRTI, with a higher risk for progression to LRTI seen across several viruses (Supplementary Fig. 3). However, trends are less clear for certain viruses with smaller cohorts.

Since hyperglycemia is associated with steroid use and higher doses of steroid use is a well-known risk factor disease progression in $\mathrm{HCT}$ recipients $[3,19,27]$, we attempted to assess the effect of hyperglycemia independently from steroid dose. Overall, our models showed a positive association between hyperglycemia and LRTI outcomes. However, the trend was not observed for the high steroid dose group. Although an effect of hyperglycemia independent from systemic steroids may be present, the relationship is not clear and it is possible that its impact on LRTI outcome differs by type of virus. Further studies could more fully assess additional effects of hyperglycemia.

We also determined which viruses were more likely to progress to LRTI at URTI stage using sensitive molecular diagnostics. Previous manuscripts have summarized progression rates for each virus [28-30]. However, progression rate data are derived primarily from past studies where differences in LRTI outcome definitions, risk factors, and treatment and diagnostic approaches were present. Because previous data may come from the premolecular diagnostic era, some viruses and particularly coinfections were likely not fully assessed. We have been consistently utilizing multiplex PCR for clinically relevant respiratory viruses for over a decade, allowing us to more consistently identify LRTI risk factors. Our observations are consistent with previously reported progression rates by virus type. In immunocompetent children, there is not convincing evidence that respiratory viral copathogens are associated with increased risk of disease severity [31, 32]. It is possible that an immune response (antiviral cytokines such as interferon) to one virus could modify the disease severity of the other virus [32], but it is unclear whether detection of multiple viruses at URTI stage is more or less likely to progress to LRTI in immunocompromised hosts. Based on our multivariable models, $\mathrm{HCT}$ recipients with multiple viruses detected to have a similar probability of progression to LRTI compared to those with only HRV, PIV, influenza, or adenovirus detected.

We also described the incidence rate of LRTI after first infection by each virus type in this large cohort. The incidence of HRV LRTI surpassed that of LRTI due to other viruses. This observation has been little appreciated [28, 29,33]. The disease burden of HRV in this vulnerable population is worthy of comprehensive assessment in this molecular diagnostic era $[27,34]$.

ISI was originally developed to predict progression to LRTI among transplant recipients presenting with RSV URTI [4]. The score has been evaluated for other viruses (e.g., influenza) where the analyses did not differentiate between patients who presented with LRTI vs. those who presented with URTI and then progressed to LRTI [5-10]. Assessing risk factors for progression at URTI stage is informative for clinical care with consideration of using potentially toxic antiviral agents (e.g., ribavirin, cidofovir) and design of early intervention clinical trials from a risk stratification perspective. In this current report, patients with a high ISI group are seen to be generally at higher risk for progression to LRTI. The trends appear stronger for RSV, HRV, PIV, influenza, and adenovirus. Regarding findings for other viruses, it is unclear whether different degree of associations between those risk factors and LRTI outcomes by virus exists, or whether our results indicate lack of power for subjects within certain virus groups. Despite the large cohort evaluated in this study, our sample size does not allow us to perform deeper analysis to tease out those possibilities.

We also estimated the probability of LRTI progression for each virus type according to number of risk factors at URTI stage. This simplified scoring system has been previously proposed for 
respiratory disease progression due to PIV [35]. This handy tool appears to perform similarly well to ISI. Patients with $>=3$ risk factors are more likely to progress to LRTI and the results are broadly consistent for all virus types except for the group with $>1$ viruses. This may be due to the clinical heterogeneity within this group. Further studies are needed to assess whether our risk stratification model with relatively simple variables can predict progression to LRTI in other cohorts.

An important finding of this study is that body habitus was not associated with increased risk of progression to LRTI. Body habitus, especially obesity, has been reported as a risk factor for severe respiratory virus infections [36-39]. This has not been well evaluated in HCT recipients; our results imply other factors are more important in this highly complex and vulnerable population. In addition, different types of endpoint in other populations may explain the inconsistent findings.

This study has several limitations. Despite the large cohort of allogeneic HCT recipients, the sample size was nonetheless not large enough to perform multivariable models to fully assess independent effects of hyperglycemia on LRTI progression from steroid dose as well as assess the degree to which associations between risk factors and LRTI outcome differ by virus. Similarly, the effect of antiviral therapy was not evaluated due to small numbers. In addition, the timing of antiviral therapy, as well as the efficacy of each therapy, differ by virus. Lastly, given the nature of retrospective studies, we cannot rule out the possibility of unrecognized confounders.

In conclusion, our study significantly improves the ability to identify patients at risk for progression to LRTI when presenting with URTI with novel risk factors including history of multiple transplants and hyperglycemia, providing a potential intervention opportunity including glycemic control. Risk stratification using accessible variables can be useful for clinical management and clinical trials in the current molecular diagnostic era.

\section{REFERENCES}

1. Chemaly RF, Shah DP, Boeckh MJ. Management of respiratory viral infections in hematopoietic cell transplant recipients and patients with hematologic malignancies. Clin Infect Dis. 2014;59:S344-51.

2. Pochon C, Voigt S. Respiratory virus infections in hematopoietic cell transplant recipients. Front Microbiol. 2018;9:3294.

3. Waghmare A, Englund JA, Boeckh M. How I treat respiratory viral infections in the setting of intensive chemotherapy or hematopoietic cell transplantation. Blood. 2016;127:2682-92.

4. Shah DP, Ghantoji SS, Ariza-Heredia EJ, Shah JN, El Taoum KK, Shah PK, et al. Immunodeficiency scoring index to predict poor outcomes in hematopoietic cell transplant recipients with RSV infections. Blood. 2014;123:3263-8.

5. Kmeid J, Vanichanan J, Shah DP, El Chaer F, Azzi J, Ariza-Heredia EJ, et al. Outcomes of influenza infections in hematopoietic cell transplant recipients: Application of an Immunodeficiency Scoring Index. Biol Blood Marrow Transpl. 2016;22:542-8.

6. Pinana JL, Xhaard A, Tridello G, Passweg J, Kozijn A, Polverelli N, et al. Seasonal human coronaviruses respiratory tract infection in recipients of allogeneic hematopoietic stem cell transplantation. J Infect Dis. 2020;223:1564-75.

7. Wang L, Allen J, Diong C, Goh YT, Gopalakrishnan S, Ho A, et al. Respiratory virus infection after allogeneic hematopoietic stem cell transplant in a tropical center: Predictive value of the immunodeficiency scoring index. Transpl Infect Dis. 2017;19:e12693.

8. Pinana JL, Hernandez-Boluda JC, Calabuig M, Ballester I, Marin M, Madrid S, et al. A risk-adapted approach to treating respiratory syncytial virus and human parainfluenza virus in allogeneic stem cell transplantation recipients with oral ribavirin therapy: A pilot study. Transpl Infect Dis. 2017;19:e12729.

9. Khawaja F, Chemaly RF. Respiratory syncytial virus in hematopoietic cell transplant recipients and patients with hematologic malignancies. Haematologica. 2019;104:1322-31.

10. Vicent MG, Martinez AP, Trabazo Del Castillo M, Molina B, Sisini L, Moron-Cazalilla G, et al. COVID-19 in pediatric hematopoietic stem cell transplantation: The experience of Spanish group of transplant (GETMON/GETH). Pediatr Blood Cancer. 2020;67:e28514.
11. Cai Y, Shi S, Yang F, Yi B, Chen X, Li J, et al. Fasting blood glucose level is a predictor of mortality in patients with COVID-19 independent of diabetes history. Diabetes Res Clin Pr. 2020;169:108437.

12. Goyal A, Gupta S, Gupta Y, Tandon N. Proposed guidelines for screening of hyperglycemia in patients hospitalized with COVID-19 in low resource settings. Diabetes Metab Syndr. 2020;14:753-6.

13. Singh AK, Singh R. Hyperglycemia without diabetes and new-onset diabetes are both associated with poorer outcomes in COVID-19. Diabetes Res Clin Pr. 2020;167:108382.

14. Ogimi C, Xie H, Waghmare A, Ueda Oshima M, Mallhi KK, Jerome KR, et al. Risk factors for seasonal human coronavirus lower respiratory tract infection after hematopoietic cell transplantation. Blood Adv. 2021;5:1903-14.

15. Campbell AP, Guthrie KA, Englund JA, Farney RM, Minerich EL, Kuypers J, et al. Clinical outcomes associated with respiratory virus detection before allogeneic hematopoietic stem cell transplant. Clin Infect Dis. 2015;61:192-202.

16. Kuypers J, Wright N, Corey L, Morrow R. Detection and quantification of human metapneumovirus in pediatric specimens by real-time RT-PCR. J Clin Virol. 2005;33:299-305.

17. Kuypers J, Wright N, Ferrenberg J, Huang ML, Cent A, Corey L, et al. Comparison of real-time PCR assays with fluorescent-antibody assays for diagnosis of respiratory virus infections in children. J Clin Microbiol. 2006;44:2382-8.

18. Seo S, Xie H, Campbell AP, Kuypers JM, Leisenring WM, Englund JA, et al. Parainfluenza virus lower respiratory tract disease after hematopoietic cell transplant: Viral detection in the lung predicts outcome. Clin Infect Dis. 2014;58:1357-68.

19. Seo S, Gooley TA, Kuypers JM, Stednick Z, Jerome KR, Englund JA, et al. Human metapneumovirus infections following hematopoietic cell transplantation: Factors associated with disease progression. Clin Infect Dis. 2016;63:178-85.

20. Jung SH, Jang HC, Lee SS, Ahn JS, Yang DH, Kim YK, et al. The impact of hyperglycemia on risk of severe infections during early period of induction therapy in patients with newly diagnosed multiple myeloma. Biomed Res Int. 2014:2014:413149.

21. Chemaly RF, Dadwal SS, Bergeron A, Ljungman P, Kim YJ, Cheng GS, et al. A Phase 2, Randomized, Double-blind, Placebo-controlled trial of presatovir for the treatment of respiratory syncytial virus upper respiratory tract infection in hematopoietic-cell transplant recipients. Clin Infect Dis. 2020;71:2777-86.

22. Reading PC, Allison J, Crouch EC, Anders EM. Increased susceptibility of diabetic mice to influenza virus infection: Compromise of collectin-mediated host defense of the lung by glucose? J Virol. 1998;72:6884-7.

23. Hulme KD, Gallo LA, Short KR. Influenza virus and glycemic variability in diabetes: A killer combination? Front Microbiol. 2017;8:861.

24. Xiu F, Stanojcic M, Diao L, Jeschke MG. Stress hyperglycemia, insulin treatment, and innate immune cells. Int J Endocrinol. 2014;2014:486403.

25. Wijsman CA, Mooijaart SP, Westendorp RG, Maier AB. Responsiveness of the innate immune system and glucose concentrations in the oldest old. Age (Dordr). 2012;34:983-6.

26. Wang $Q$, Fang $P$, He R, Li M, Yu H, Zhou L, et al. O-GlcNAc transferase promotes influenza $A$ virus-induced cytokine storm by targeting interferon regulatory factor-5. Sci Adv. 2020;6:eaaz7086.

27. Waghmare A, Xie H, Kuypers J, Sorror ML, Jerome KR, Englund JA, et al. Human rhinovirus infections in hematopoietic cell transplant recipients: Risk score for progression to lower respiratory tract infection. Biol Blood Marrow Transplant. 2018;25:1011-21.

28. Shahani L, Ariza-Heredia EJ, Chemaly RF. Antiviral therapy for respiratory viral infections in immunocompromised patients. Expert Rev anti-infective Ther. 2017;15:401-15.

29. Renaud C, Englund JA. Antiviral therapy of respiratory viruses in haematopoietic stem cell transplant recipients. Antivir Ther. 2012;17:175-91.

30. Hirsch HH, Martino R, Ward KN, Boeckh M, Einsele H, Ljungman P. Fourth European Conference on Infections in Leukaemia (ECIL-4): Guidelines for diagnosis and treatment of human respiratory syncytial virus, parainfluenza virus, metapneumovirus, rhinovirus, and coronavirus. Clin Infect Dis. 2013;56:258-66.

31. Scotta MC, Chakr VC, de Moura A, Becker RG, de Souza AP, Jones MH, et al. Respiratory viral coinfection and disease severity in children: A systematic review and meta-analysis. J Clin Virol. 2016;80:45-56.

32. Martin ET, Kuypers J, Wald A, Englund JA. Multiple versus single virus respiratory infections: Viral load and clinical disease severity in hospitalized children. Influenza Other Respir Viruses. 2012;6:71-77.

33. Pinana JL, Perez A, Montoro J, Hernani R, Lorenzo I, Gimenez E, et al. The effect of timing on community acquired respiratory virus infection mortality during the first year after allogeneic hematopoietic stem cell transplantation: A prospective epidemiological survey. Bone Marrow Transpl. 2020;55:431-40.

34. Seo S, Waghmare A, Scott EM, Xie H, Kuypers JM, Hackman RC, et al. Human rhinovirus detection in the lower respiratory tract of hematopoietic cell transplant recipients: association with mortality. Haematologica. 2017;102:1120-30. 
35. Seo S, Xie H, Leisenring WM, Kuypers JM, Sahoo FT, Goyal S, et al. Risk factors for parainfluenza virus lower respiratory tract disease after hematopoietic cell transplantation. Biol Blood Marrow Transpl. 2019;25:163-71.

36. Longmore DK, Miller JE, Bekkering S, Saner C, Mifsud E, Zhu Y, et al. Diabetes and overweight/obesity are independent, Nonadditive risk factors for in-hospital severity of COVID-19: An international, multicenter retrospective meta-analysis. Diabetes Care. 2021;44:1281-90.

37. Zhao X, Gang X, He G, Li Z, Lv Y, Han Q, et al. Obesity increases the severity and mortality of influenza and COVID-19: A systematic review and meta-analysis. Front Endocrinol (Lausanne). 2020;11:595109.

38. Moser JS, Galindo-Fraga A, Ortiz-Hernandez AA, Gu W, Hunsberger S, GalanHerrera JF, et al. Underweight, overweight, and obesity as independent risk factors for hospitalization in adults and children from influenza and other respiratory viruses. Influenza Other Respir Viruses. 2019;13:3-9.

39. Pettit NN, MacKenzie EL, Ridgway JP, Pursell K, Ash D, Patel B, et al. Obesity is associated with increased risk for mortality among hospitalized patients with COVID-19. Obes (Silver Spring). 2020;28:1806-10.

\section{ACKNOWLEDGEMENTS}

We thank Lucy Maher, Daniela Ramos, and Sydnee Dismuke for data collection and Chris Davis and Ryan Basom for database services.

\section{AUTHOR CONTRIBUTIONS}

CO designed this study, assisted in the analysis, interpreted results, and wrote the paper; HX and WML performed the statistical analysis and wrote the paper; AW, MU, and PAC provided clinical input, interpretation of results, and reviewed the paper; KRJ provided technical oversight for laboratory, and reviewed the paper; JAE and $M B$ provided oversight, designed this study, interpreted results, and reviewed the paper.

\section{FUNDING}

This work was supported by the National Institutes of Health (grant numbers K23Al139385 to CO, R01HL081595, and K24HL093294 to MB, CA18029 to WL, clinical database, CA15704 to HX); the Fred Hutchinson Cancer Research Center Vaccine and Infectious Disease Division (biorepository); Seattle Children's Research Institute Clinical Research Scholars Program Award) to CO.

\section{COMPETING INTERESTS}

AW reports grant support from Ansun Biopharma, Allovir, VB Tech, Amazon, Inc, GlaxoSmithKline and Pfizer, and is an Advisory Board Member for Kyorin Pharmaceutical. JAE reports grant support from AstraZeneca, Merck, Pfizer, GlaxoSmithKline and Novavax, is an Advisory Board Member and consultant for Sanofi Pasteur, and is a consultant for Meissa Vaccines. MB reports grant support from Amazon, GSK, Regeneron, Janssen, Gilead and VirBio; he is a consultant for Allovir, Janssen, Gilead, ReViral and VirBio, and an Advisory Board Member for Evrys Bio (option to purchase shares). All other authors report no potential conflicts. Conflicts that the editors consider relevant to the content of the manuscript have been disclosed.

\section{ADDITIONAL INFORMATION}

Supplementary information The online version contains supplementary material available at https://doi.org/10.1038/s41409-022-01575-z.

Correspondence and requests for materials should be addressed to Chikara Ogimi.

Reprints and permission information is available at http://www.nature.com/ reprints

Publisher's note Springer Nature remains neutral with regard to jurisdictional claims in published maps and institutional affiliations. 